\title{
Exploration of Adaptive Beaconing for Efficient Intervehicle Safery Communication
}

\author{
Robert K. Schmidt and Tim Leinmüller, DENSO AUTOMOTIVE \\ Elmar Schoch and Frank Kargl, Ulm University \\ Günter Schäfer, Technische Universität Ilmenau
}

\begin{abstract}
In the future intervehicle communication will make driving safer, easier, and more comfortable. As a cornerstone of the system, vehicles need to be aware of other vehicles in the vicinity. This cooperative awareness is achieved by beaconing, the exchange of periodic single-hop broadcast messages that include data on the status of a vehicle. While the concept of beaconing has been developed in the first phase of research on VANETs, recent studies have revealed limitations with respect to network performance. Obviously, the frequency of beacon messages directly translates into accuracy of cooperative awareness and thus traffic safety. There is an indisputable trade-off between required bandwidth and achieved accuracy. In this work we analyze this trade-off from different perspectives considering the consequences for safety applications. As a solution to the problem of overloading the channel, we propose to control the offered load by adjusting the beacon frequency dynamically to the current traffic situation while maintaining appropriate accuracy. To find an optimal adaptation, we elaborate on several options that arise when determining the beacon frequency. As a result, we propose situation-adaptive beaconing. It depends on the vehicle's own movement and the movement of surrounding vehicles, macroscopic aspects like the current vehicle density, or microscopic aspects.
\end{abstract}

ne of the major goals of vehicular ad hoc networks (VANETs) is to support traffic safety. Cooperative awareness applications require frequent and low-delay information exchange among vehicles, including data such as current position, movement, and acceleration. This is realized by broadcasting socalled (single-hop) beacon messages. As a result, every vehicle is aware of other vehicles within a certain range. Beaconing is also the basic supporting process that enables geographic routing and message dissemination. However, this also requires a significant amount of bandwidth. The higher the frequency and thus the accuracy, the higher the bandwidth consumption.

The first phase of research on VANETs has set the boundary conditions in terms of basic communication protocols and routing paradigms. Communication in VANETs will be based on IEEE 802.11p. Message dissemination and routing are based on geocast principles. Beaconing takes place on a single communication channel (commonly referred to as the control channel) that is shared by all nodes.

However, it has also been shown in [1] that the limited bandwidth of the wireless channel has a severe impact on the efficiency of the communication. This means that if the beacon rate is fixed, channel load may increase too much in scenarios with high vehicle density. High channel utilization increases the information loss as packets are received erroneously, which is especially observed at large distances between sender and receiver.
Simply reducing the beacon rate is not a suitable solution because it reduces the information quality at the same time. The error between the real position of a vehicle and the last known position retrieved from a beacon increases as the beacon rate is reduced. This results in position inaccuracies, which may disturb correct operation of active safety applications, which rely on accurate and up-to-date information.

Currently, there is no final recommendation for a particular static beacon rate. No upper boundary in terms of maximum channel load has been specified. Furthermore, no requirements from the different applications have yet been clearly defined. As a consequence, even minimum and maximum beacon rate are hard to derive as these two boundaries have to satisfy all imaginable road traffic situations.

In this article we pave the way for further enhancements of beaconing algorithms for the second phase of research on VANETs. After a short assessment of the current state of the art, we present a detailed analysis of the problem space and how different beacon rates influence 1) the offered load to the channel and 2) the resulting average and maximum accuracy of information

Based on this problem evaluation, we motivate a flexible approach to control both appropriately. For this purpose, we propose a situation adaptive beaconing process that adapts the beacon rate continuously. The design space and different candidates for such an algorithm are introduced in this article. 
The difficulties with this design are the two optimization goals which seem to be conflicting at the first glance:

- Low resource usage: Less occupancy of the wireless medium is achieved by reducing the beacon rate. It is better to receive less information but still reliably than to have unpredictable high packet loss.

- High information accuracy: The knowledge of the surrounding vehicles must be as accurate and up-to-date as possible, which can be achieved with a higher beacon rate.

In order to evaluate our concepts, we analyze the influence of different beacon rates on the channel load and achievable accuracy. Therefore, we denote the offered load as the number of beacons sent by all vehicles in relation to the channel capacity. For determining the accuracy of a particular beacon rate, we introduce the position accuracy metric where we consider the error between the current physical position and the last reported position.

\section{Related Work}

The adaptation of beaconing may cover different aspects in general. Widely discussed are parameters such as the transmit power (e.g., [2]) or beacon rate; the latter is addressed in this article. As we focus on the impact of adaptation on the accuracy of information, in the following we look at related work in the area of beacon rate adaptation.

In [3] Khorakhun et al. propose that all nodes should adapt the beacon rate depending on the current channel load, measured via the channel busy ratio. The goal is to let the offered load converge to a given maximum allowed channel load. To achieve a smooth adaptation, an average beacon rate is calculated and exchanged among the vehicles. Each vehicle then adapts its beacon frequency to the average.

Another direction of adapting the beacon rate is taken by Rezaei et al. in $[4,5]$ where they present a concept to adapt the beaconing rate depending on differences from position predictions. They assume that all vehicles run the same position prediction algorithm. An extended Kalman filter is locally applied to each vehicle in the neighbor table. It continuously estimates the current position based on the received position information history in order to improve the accuracy of the position during the time between two successive beacons. The time to send the next beacon is determined based on the following algorithm. The vehicle knows its own physical position and is able to estimate its own position the same way all surrounding vehicles do. Once it determines that its own physical position has a particular difference from the remote estimator, it sends the next beacon. This approach is a suitable concept to inform neighboring vehicles about movement changes. However, there are several drawbacks with the presented approach when thinking about active safety applications. Position prediction is critical because active safety applications will become active in situations where the movement of a vehicle changes suddenly, and thus the prediction is inaccurate. Also, it does not account for situations where higher beacon rates are needed, such as in case of an imminent collision (but no change of movement). Another problem is that once an application decision whether to warn the driver has to be made, the current prediction error is not known to the receiver. Furthermore, message loss and frequent network topology changes are not treated; for example, the defined maximum error can be exceeded due to message loss. A countermeasure would be, for instance, to introduce a minimum beacon rate.

A step toward situation-adaptive beaconing was made by Fukui et al. in [6]. Beacons are sent periodically based on a constant distance a vehicle has to travel. Furthermore, each vehicle determines the current number of lanes. For multilane roads, the beacon rate is reduced. Basically, with this approach situations with potentially high node densities are detected, and hence the beacon rate is reduced appropriately. A further adaptation is applied by considering the current packet error rate. However, the defined goal is only to reduce the offered load and the number of colliding packets. The effect of reduced accuracy has been neglected, which may cause problems, for example, at multilane intersections.

In summary, each approach addresses only one optimization goal. The approach of Khorakhun et al. prevents overloading of the channel but does not account for maintaining position accuracy. Rezaei et al. do not consider the node density nor do they react on the information they receive from surrounding vehicles. Finally, Fukui et al. neglect the effect of reduced accuracy.

\section{Adaptive Beaconing in VANETs}

As mentioned in the introduction, we consider both goals for beaconing, to reduce offered load and provide the best possible information accuracy for safety applications and routing. Therefore, we propose to adapt the beacon rate according to the following requirements and influences:

- Offered load limitation: Beaconing may be the application consuming most of the bandwidth. It must be ensured that the channel does not get overloaded since it would lead to significant decrease of performance in terms of high packet error rates.

- Minimum of available neighbor information: A minimum beacon rate must be maintained to ensure the discovery of new neighbors with low delay and keep the neighbor table up to date.

- Appropriate resolution: A beaconing frequency of $10 \mathrm{~Hz}$ is not necessary in a traffic jam with vehicles standing still, whereas $1 \mathrm{~Hz}$ may be not sufficient on a multilane highspeed highway with frequent lane changes. Thus, the maximum time interval and maximum driving distance between two beacons must be limited. As a traffic situation also depends on the movement of the surrounding vehicles, the adaptation of the beacon rate must consider position, speed, acceleration, direction, and yaw rate of surrounding vehicles.

These requirements are to be considered for the problem evaluation as well as the design of adaptive beaconing concepts.

\section{Adaptation Evaluation Criteria}

We discuss selected beacon rates based on desired accuracy and offered load. Our discussion is guided by the following questions:

- What is an appropriate beacon rate at a particular velocity for a desired accuracy?

- Which beacon rate is appropriate at a particular node density to keep a desired maximum offered load?

For the evaluation of different beacon rates, we introduce the position accuracy metric as a measure for the accuracy of a particular beacon rate.

\section{Metric for Position Accuracy}

We define the metric to reflect three criteria: the minimum error, maximum error, and average error of the last received position information in relation to the current physical position of a vehicle. The relevant input parameters for the metric are the vehicle velocity $v$, beacon rate $f_{B}$ and transmission delay $t_{T x}$. The resulting accuracy metric is as follows: 


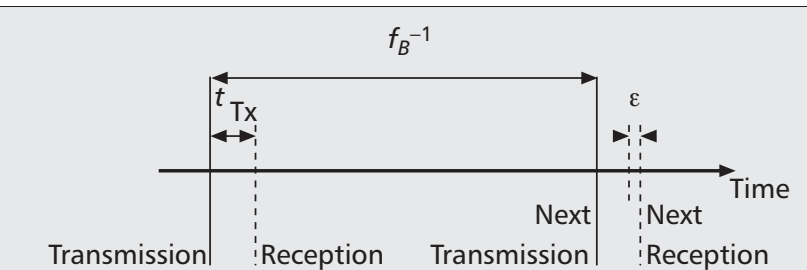

Figure 1. Relation of relevant time parameters that determine the accuracy.

- Minimum position error $\lfloor E\rfloor$ : denotes the lower error boundary resulting from the transmission delay $t_{T x}$. The minimum position error is usually negligible as the transmission delay $t_{T x}$ is typically around $0.001 \mathrm{~s}$ and thus relatively small compared to the lowest beacon interval of $0.1 \mathrm{~s}$.

- Maximum position error $\lceil E\rceil$ : is the upper boundary that occurs when the position of a vehicle is looked up $(\in)$ right before receiving the next beacon from this vehicle. This error is equal to the distance the vehicle travels during a beacon interval minus a small time value $\epsilon$.

- Average position error $\bar{E}$ : expresses the mean error assuming that the event of looking up the position is uniformly distributed between minimum and maximum time difference to the transmission event of the beacon. Figure 1 sketches the time dependencies of the metric. The corresponding equations can easily be derived as

$$
\bar{E}=\frac{\lfloor E\rfloor+\lceil E\rceil}{2}=v t_{T x}+\frac{v\left(f_{B}^{-1}-\varepsilon\right)}{2} .
$$

In the following evaluation we focus on the discussion of the maximum and average errors. We do not discuss the minimum error as it solely depends on the transmission delay. As the parameters have wide ranges (e.g., velocity), and may not have been clearly defined (e.g., the beacon rate), we define typical values for the remaining analysis and discussion.

The velocity of a vehicle may vary in a range of 0 to 250 $\mathrm{km} / \mathrm{h}$, depending on many factors and conditions. We select four values for maximum speed, which can be observed in four different traffic scenarios: residential areas $(30 \mathrm{~km} / \mathrm{h})$, metropolitan areas $(50 \mathrm{~km} / \mathrm{h})$, rural roads $(100 \mathrm{~km} / \mathrm{h})$, and highways $(200 \mathrm{~km} / \mathrm{h})$.

Note that for the sake of simplicity, we assume constant speed without acceleration or deceleration during two successive beacons. Also, changes in the direction of a vehicle are not considered. The possible change of movement would only provide for a small offset given by maximum acceleration and yaw rate. For the special case of $v=0$, the error would be 0 .

Commonly discussed are beacon rates $f_{B}$ between 1 and 10 beacons/s. For our discussion we therefore investigate four typical values: high $(10 \mathrm{~Hz})$, medium $(5 \mathrm{~Hz})$, low $(2 \mathrm{~Hz})$, and minimum $(1 \mathrm{~Hz})$ beacon rate.

\section{Accuracy Evaluation}

Figure $2 \mathrm{a}$ visualizes the influence of different beacon rates and vehicle velocities on the average position error calculated according to Eq. 1 as introduced and explained in the previous section. Each graph shows a particular error level, ranging from $1 \mathrm{~m}$ up to $10 \mathrm{~m}$ average position error, reflecting high down to low accuracy. We make the following observations. With high beacon rate of 10 beacons/s, an accuracy of $1 \mathrm{~m}$ is maintained up to a velocity of about $70 \mathrm{~km} / \mathrm{h}$. An accuracy of $10 \mathrm{~m}$ is achieved with low beacon rate up to a velocity of about $150 \mathrm{~km} / \mathrm{h}$. At high speed $(200 \mathrm{~km} / \mathrm{h})$, the medium beacon rate of 5 beacons/s has to be applied to stay at within 10 $\mathrm{m}$ of average error.

The minimum beacon rate provides an accuracy of $5 \mathrm{~m}$ only at low velocities $(30 \mathrm{~km} / \mathrm{h})$, whereas for a metropolitan area with $50 \mathrm{~km} / \mathrm{h}$, only an accuracy of $10 \mathrm{~m}$ is met.

The discussion of the maximum error is similar. As shown in Eq. 1, the maximum error is twice the average error. Hence, the graph for $1 \mathrm{~m}$ average error is the same for the maximum error of $2 \mathrm{~m}$. The graph of $5 \mathrm{~m}$ average error corresponds to the graph of $10 \mathrm{~m}$ maximum error.

\section{Offered Load Evaluation}

For the discussion of the offered load, we make the following assumptions and simplifications. We assume $6 \mathrm{Mb} / \mathrm{s}$ as data rate on the communication channel, in line with research from Jiang et al. [7]. Furthermore, we consider only the percentage of the offered load (payload) in relation to the (gross) data rate where we neglect any (varying) overhead by the medium access control (MAC) and physical (PHY) layers (e.g., headers, contention window, and interframe spaces).

In Fig. $2 b$ we show two levels of offered load with a globally applied beacon rate. As packet sizes have not been fixed or limited yet, we distinguish two packet lengths: medium-size packets of 200 bytes each and large packets of 1000 bytes each. The main reason for considering large packets is that beacons might contain overhead from cryptographic signatures and key material. The results for small packets ( 50 bytes) are not shown as for the given constraints of desired offered load, number of vehicles in communication range, and beacon rate, the channel capacity is not exceeded.

The two offered loads are chosen according to [8], where Brakemeier argues that for efficient channel usage the offered load should be between 40 and 60 percent. According to his work, an offered load of 60 percent plus PHY/MAC protocol overhead may fully utilize the channel.

For large packets, the desired offered loads are reached at 300 and 450 vehicles even with the minimum beacon rate. With 10 beacons/s, only 50 and 90 vehicles are supported at maximum. We also see only a slight difference for 40 and 60 percent.

The situation relaxes at medium packet size. For example, 300 vehicles in communication range and a beacon rate of 5 beacons/s result in 40 percent offered load. Likewise, with 10 beacons/s 150 vehicles are supported for that load. Still, with higher densities the offered load can increase to more than 60 percent.

These examples show the limitations of efficient communication in the particular scenarios. High beacon rates cannot be supported for all vehicles in cases of high vehicle densities or larger packets.

\section{Accuracy vs. Offered load}

The previous discussion on accuracy considers the accuracy of position information of a single vehicle when using a certain beacon rate. In the discussion of the offered load, we have considered all vehicles within communication range as they all contribute with their beacon transmissions. Setting both into relation does not provide sufficient criteria for beacon rate adaptation. Basically, one can derive the offered load for a given average speed (of all vehicles) and a particular beacon rate. In relation to per-vehicle accuracy, the achieved (average) accuracy can be estimated.

However, the heterogeneity of velocities should be taken into account in the discussion of beacon rate adaptation. Imagine a traffic jam with vehicles driving slowly at the same velocity. At a constant beacon rate, the position of these vehicles can be determined with the same accuracy. But oncoming traffic may not be jammed and travel at high speeds. For the same beacon rate, the accuracy would be much lower. For 

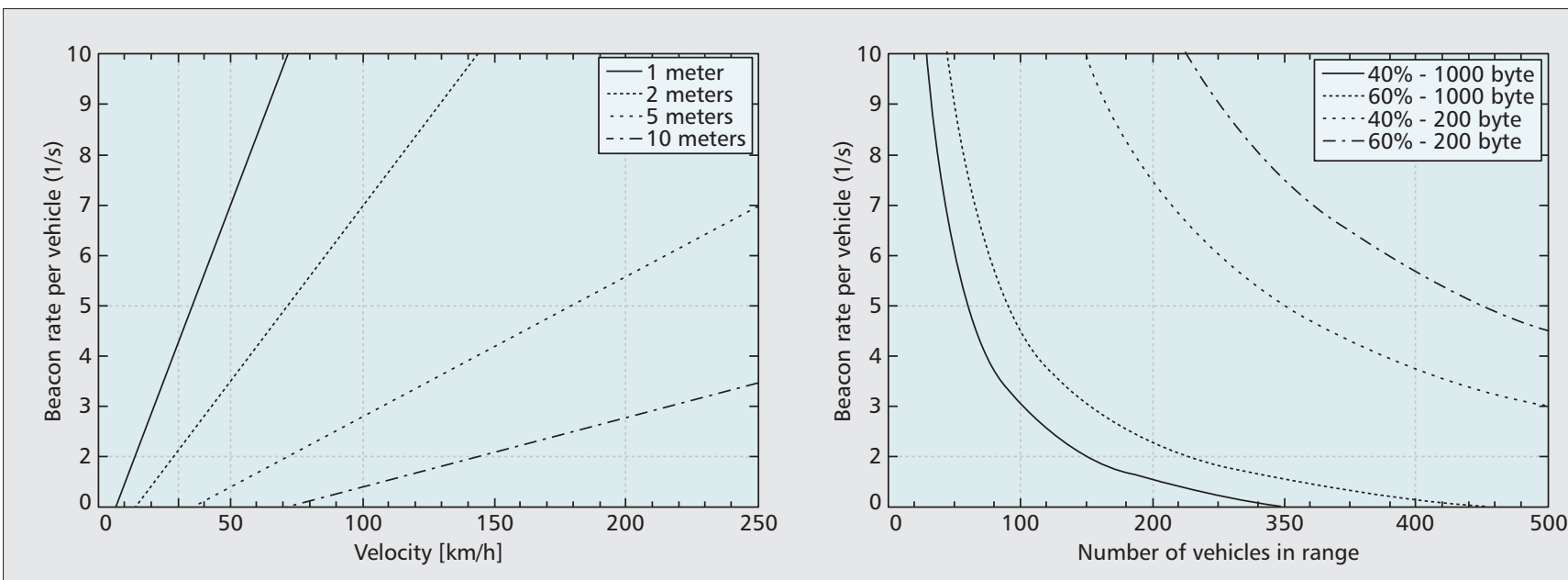

Figure 2. a) Average position error depending on beacon rate and velocity, b) amount of data produced (offered load) by beaconing depending on beacon rate and number of vehicles in communication range.

these vehicles a much higher beacon rate is required to meet the same accuracy requirements. This could result in situations where the overall offered load exceeds the channel capacity.

With this example we want to highlight that there are many parameters that have to be considered; especially their sophisticated interrelation has to be taken into account in the design of adaptive beaconing. Furthermore, the difference of changing the beacon frequency globally or only locally in individual vehicles must be investigated further. Summarizing, the beacon rate should be adapted based on a vehicle's current context, which we denote as situation-adaptive beaconing.

\section{Schemes for Situation-Adaptive Beaconing}

Situation-adaptive beaconing basically depends on the vehicle's own status and the road traffic situation in consideration with the currently offered load. Accordingly, we discuss two categories of schemes for rate adaptation, depending on the vehicle's own movement and depending on surrounding vehicles' movement. Schemes that act depending on a vehicle's own movement are able to adapt the beacon rate based on the vehicle's status to maintain a defined accuracy. These schemes do not use any information from other vehicles. This, in turn, is the case for the second category of adaptation schemes. They either depend on characteristics of the whole traffic situation (macroscopic view) or adapt the rate with respect to particular situations (microscopic view). Figure 3 gives an overview of the different schemes. For each of them, we discuss the impact on accuracy and offered load.

\section{Adaptation Depending on a Vehicle's Own Movement}

This adaptation considers solely the current status of the transmitting vehicle. According to Fig. 3, there are three main criteria that can be taken into account. The first criterion is the velocity of a vehicle. As we have already discussed earlier, the accuracy strongly depends on the current velocity. Thus, the beacon rate should be higher for higher velocities, by either linearly increasing the beacon rate $f_{B}$ or changing it in specified steps. Especially to maintain the maximum offered load, an additional criterion may be needed. The resulting offered load is implicitly controlled by the relationship between average velocity and traffic density. This fundamental relationship is commonly known from traffic flow theory; for example, in [9] Kerner states that for increasing traffic density, the achievable average speed decreases. As a result, the offered load remains stable for more vehicles driving at lower velocities. Nevertheless, additional criteria are required to maintain the maximum offered load.

For adaptation based on movement changes we basically share the opinion of Rezeai et al. [4,5] that situations where a vehicle changes its movement have a higher potential for dangerous situations. For example, heavy braking, turning, or changing a lane are events where accidents may occur. Their approach implicitly considers all movement parameters. We propose to consider all parameters explicitly. According to Fig. 3, movement changes can be divided into acceleration (and deceleration) and yaw rate. Optionally, vehicle-internal sensors may trigger a higher beacon rate (e.g., in case of reduced friction).

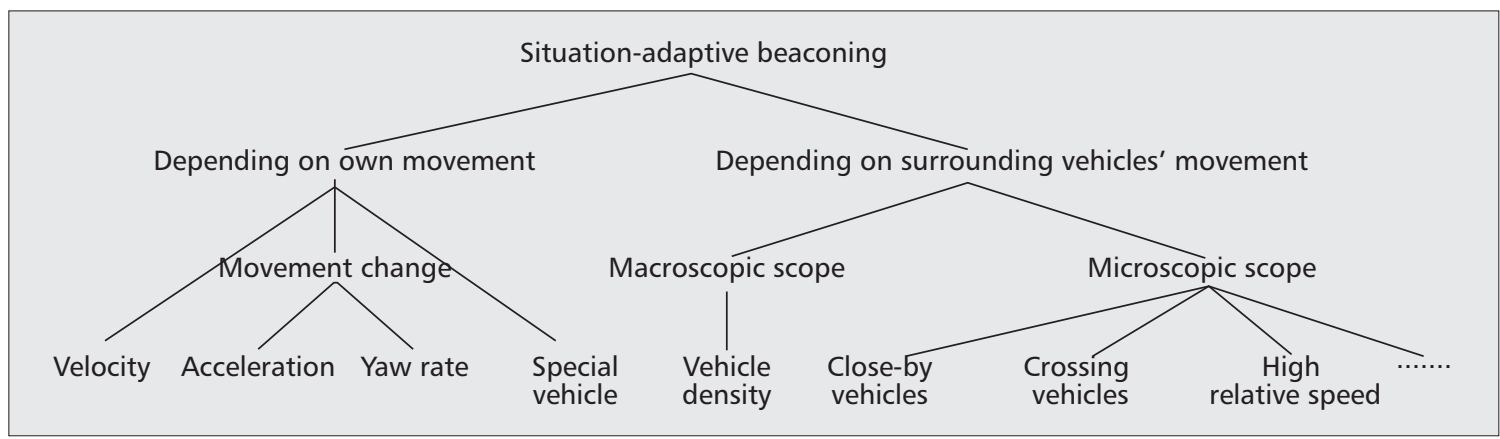

Figure 3. Overview on essential schemes for situation-adaptive beaconing. 
The last criterion covers vehicles with special movement patterns, such as emergency vehicles. They may have an increased beacon rate as their position information is assumed to have higher priority, informing other vehicles to clear the way, with the additional demand of high accuracy.

\section{Adaptation Depending on Surrounding Vehicles' Movement}

The schemes that are discussed in this section are based on knowledge of surrounding vehicles. They are essential to consider the situation around a vehicle. The beacon rate may be adapted by macroscopic aspects like the current vehicle density or by microscopic aspects. For example, if there is a vehicle close by due to a lane change, the beacon rate may be increased for accuracy reasons.

Macroscopic Traffic Situation - Situations with high vehicle densities may constitute a problem for reliable communication as the channel capacity may be exceeded. To proactively reduce the offered load, the beacon rate should be lowered in case the vehicle density is high. Reduction of the beacon rate increases the reliable reception of the reduced offered load. As discussed earlier, usually the beacon rate is already reduced when velocity-based adaptation is applied. This scheme additionally adapts the rate, proactively avoiding channel overload in case velocity-based is not sufficient. As a consequence, a reduction of the beacon rate by this scheme may lead to significant degradation of accuracy. The benefit would be to maintain the defined maximum offered load (i.e., keeping the communication system in a stable state).

Another approach would be to reduce the beacon rate based on the number of messages received per second instead of the number of (communicating) vehicles. The problem here is the potentially uneven distribution of beacon rates at different vehicles. Assuming vehicle A increases the rate, vehicle B would react to this with a reduction of the beacon rate, potentially leading to even further increase of the beacon rate by vehicle A. Hence, the vehicle-density-based scheme should be applied as an open-loop approach. The adaptation should not depend on the influence of the last adaptation.

Microscopic Traffic Situation - Schemes that consider microscopic aspects allow for wider adaptation of the beacon rate than the schemes described in the previous subsection. The following schemes address a few vehicles in particular situations. Hence, the impact on the offered load is small compared to macroscopic schemes where all vehicles react in parallel. Their influence is in order of the number of all vehicles within range.

Microscopic reactions trigger an increase of the beacon rate in special situations according to use cases or the requirements of currently running applications. An overview on many use cases can be found in the use case catalog of European Telecommunications Standards Institute (ETSI) TR 102638 [10]. Due to the huge number of use cases, we focus on the explanation of the key ideas for these schemes.

Vehicles that are close to each other should increase the beacon rate as the probability of collision becomes higher the closer the vehicle gets. Furthermore, two vehicles potentially crossing their ways demand a higher beacon rate. This occurs in situations like lane changes, intersections, a wrong-way driver, or vehicles at high velocities approaching slow vehicles. In these situations the beacon rate may already be high as the autonomous schemes should have detected high velocity or changes in movement. If so, the beacon rate should be increased even further if vehicle collisions are likely. The respective scheme, however, must be constrained not to exceed the maximum offered load as this may occur in situations where there are many vehicles close to each other.

Note that this category of situation-specific adaptation schemes is most sophisticated due to the variety of different traffic situations. Nonetheless, it appears to also be the class of mechanisms to cover the variety of application requirements in terms of accuracy.

\section{Combination of Schemes}

The combination of several schemes from the different categories into an adaptation framework results in situation-adaptive beaconing. The adaptation of the beacon rate has to depend on the vehicle's own status in consideration of the status of its surrounding vehicles. In the framework each scheme is provided with a weight defining the total impact on the increase of the beacon rate. The outcome of all considered schemes influences the adaptation, that is, how much to increase the beacon rate additionally to the minimum beacon rate.

The combination of schemes ensures that vehicles increase the beacon rate in special (and dangerous) situations (e.g., once they detect rapidly approaching vehicles). This supports not only active safety applications but also the routing scheme, which relies on up-to-date neighbor tables for an efficient routing decision.

An example of a highway traffic situation where an emergency vehicle approaches a traffic jam already covers many schemes described in this article and highlights how to combine them. The emergency vehicle, as a special vehicle with light bar in use, travels at high velocity. Its beacon rate is set to a high value by the schemes that depend on its own movement. The vehicles in the traffic jam use the minimum beacon rate because they are driving slowly. Once the emergency vehicle approaches the traffic jam at high relative speed being close by, the beacon rates are adapted by the tail-end vehicles in the traffic jam while the emergency vehicle keeps its high beacon rate. After passing the tail-end vehicles, they set the rate to minimum again. The other vehicles in the traffic jam increase the beacon rate temporarily as the emergency vehicle approaches them and return to the minimum rate once they have been passed (i.e., once there is no longer any imminent danger).

\section{Conclusions}

Exchanging vehicle awareness information via beacon messages is important for active safety applications as well as for geographic routing. By its periodicity of transmission, a very high load may be imposed onto the wireless channel.

In this work we study the effects of adapting the beacon rate with respect to reduced accuracy and changing offered load. We identify different accuracy demands in different traffic situations. Considering both offered load and corresponding accuracy, we analyze the spectrum of proposed beacon rates. To account for this, we proposed schemes for adapting the beacon rate according to the traffic situation. Requirements for minimum and maximum beacon rate are introduced which define the boundaries for the adaptation process. Schemes for this adaptation have been categorized by their scope. We identify the microscopic traffic-situation-based adaptation as a promising scheme to fulfill application requirements in special situations. When vehicles detect a dangerous situation, these few vehicles temporarily increase their beacon rate. The advantage of this is only a slight increase in channel load with the benefit of high accuracy by frequent position and movement updates for these affected vehicles. 
For a final situation-adaptive beaconing scheme, we propose to combine the outlined schemes in a framework that allows the aggregation of the various schemes. The aggregation result increases the beacon rate dynamically, starting from the minimum required rate. This framework should also consider weighting of the schemes, where microscopic trafficsituation-based adaptation has the greatest impact.

The adaptation of the beacon rate has been discussed to control the offered load. However, there are more techniques that can be considered. The communication channel load can be reduced by setting a lower transmit power for vehicles with temporary high beacon rates. Again, to meet the minimum requirement for geographic routing, the minimum beacon rate must be maintained at standard transmit power.

Future research work comprises a lot of challenging aspects based on beacon rate adaptation. All imaginable road traffic situations and network loads have to be covered by suitable adaptation schemes in order to provide potential life saving information to drivers. Therefore, research on smart communication has to be combined with traffic flow aspects. Applications for safety, in turn, need to define their accuracy requirements for particular situations. Correspondingly, an additional metric measuring the potential danger of a situation is needed.

\section{References}

[1] R. K. Schmidt et al., "Degradation of Transmission Range in VANETs caused by Interference," Praxis der Informationsverarbeitung und Kommunikation, Special Issue on Mobile Ad Hoc Networks, no. 4, 2009.

[2] M. Torrent-Moreno et al., "Vehicle-to- Vehicle Communication: Fair Transmi Power Control for Safety-Critical Information," IEEE Trans. Vehic. Tech., vol. 58 , no. 7, 2009, pp. 3684-703.

[3] C. Khorakhun, H. Busche, and H. Rohling, "Congestion Control for VANETs based on Power or Rate Adaptation," Wksp. Intelligent Transportation 2008, Mar. 2008.

[4] S. Rezaei et al., "Adaptive Communication Scheme for Cooperative Active Safety System," WoCo, 2008.

[5] -, "Reducing the Communication Required by DSRC-based Vehicle Safety Systems," VANET '07: Proc. 4th ACM Int'l. Wksp. Vehic. Internetworking, ACM, Sept. 2007.

[6] R. Fukui, H. Koike, and H. Okada, "Dynamic Integrated Transmission Contro (DITRAC) Over Inter-Vehicle Communications in ITS," IEEE VTC Spring 2002, vol. 1, 2002.
[7] D. Jiang, Q. Chen, and L. Delgrossi, "Optimal Data Rate Selection for Vehicle Safety Communications," VANET '08: Proc. 5th ACM Int'l. Wksp. Vehic. Internetworking, New York, NY, 2008, pp. 30-38.

[8] A. Brakemeier, "C2C-CC White Paper on Network Design Limits," C2008.

[9] B. S. Kerner, The Physics of Traffic, Empirical Freeway Pattern Features, Engineering Applications, and Theory, Springer Complexity Series: Understanding Complex Systems, Berlin, Heidelberg, New York: Springer, 2004.

[10] ETSI TR 102 638, 2009.

\section{Biographies}

ROBERT KARL SCHMIDT (r.schmidt@denso-auto.de) received his Diploma degree in computer science in 2008 from the Technical University Ilmenau, Germany. Afterward, he joined DENSO AUTOMOTIVE Deutschland GmbH where he focuses his research activities on efficient communication and security in vehicular ad hoc networks.

TIM LEINMÜLLER (t.leinmueller@denso-auto.de) received his joint degree in electrical engineering from ENST-Paris and the University of Stuttgart in 2003. Afterward, he worked for DaimlerChrysler AG Group Research and Advanced Engineering. In 2007 he joined DENSO AUTOMOTIVE Deutschland GmbH, where his activities focus on research and standardization in the area of car-tocar communication.

ELMAR SCHOCH (elmar.schoch@uni-ulm.de) received his doctorate degree in computer science from Ulm University in 2009, working on robust and efficient security mechanisms for communication in intervehicle networks. Both for Ulm University and DaimlerChrysler Telematics Research, he was involved in several European IVC projects, such as SEVECOM, PRECIOSA, and NOW. His current research interests center around advanced techniques for vehicular networks, trust, security, and privacy for mobile and ubiquitous systems.

FRANK KARGL (frank.kargl@uni-ulm.de) is an associate professor at the University of Twente in the Distributed and Embedded Security Group. Until 2009 he was a senior researcher at Ulm University, leading a research team focusing on various aspects of VANET communications including information dissemination, applications, and security and privacy. He has co-authored over 80 peer-reviewed publications, and is actively involved in research projects like $\mathrm{SeVeCom}$ and PRECIOSA. He also contributes to standardization activities, is a regular member of program committees, a reviewer for selected journals, and a panelist and keynote speaker on ITS security and privacy.

GÜNTER SCHÄFER [M] (guenter.schaefer@tu-ilmenau.de) is a full professor of telecommunications/computer networking at the University of Ilmenau, Germany. His main subject areas are network security, protection of communication infrastructures, as well as communication protocols and architectures. He is a member of the Association for Computing Machinery (ACM) and the German Gesellschaft für Informatik (Computer Science Society). 